\section{BMJ Open Respiratory Research}

\title{
Stability in eosinophil categorisation during subsequent severe exacerbations of COPD
}

\author{
Emanuel Citgez, ${ }^{1,2}$ Job van der Palen, ${ }^{2,3}$ Paul van der Valk, ${ }^{1}$ Huib A M Kerstjens, ${ }^{4}$ \\ Marjolein Brusse-Keizer ${ }^{3}$
}

\begin{abstract}
To cite: Citgez $\mathrm{E}$, van der Palen J, van der Valk P, et al. Stability in eosinophil categorisation during subsequent severe exacerbations of COPD. BMJ Open Resp Res 2021;8:e000960. doi:10.1136/ bmjresp-2021-000960

- Additional supplemental material is published online only. To view, please visit the journal online (http://dx.doi. org/10.1136/bmjresp-2021000960).
\end{abstract}

Received 19 April 2021 Accepted 27 June 2021

Check for updates

(C) Author(s) (or their employer(s)) 2021. Re-use permitted under CC BY-NC. No commercial re-use. See rights and permissions. Published by BMJ.

${ }^{1}$ Department of Pulmonary Medicine, Medisch Spectrum Twente, Enschede, The Netherlands

${ }^{2}$ Department of Research Methodology, Measurement, and Data analysis, University of Twente, Enschede, The Netherlands

${ }^{3}$ Medical School Twente, Medisch Spectrum Twente, Enschede, The Netherlands ${ }^{4}$ Department of Pulmonary Medicine, and Groningen Research Institute for Asthma and COPD (GRIAC), University of Groningen, University Medical Centre Groningen, Groningen, The Netherlands

Correspondence to Dr Emanuel Citgez; e.citgez@mst.nl

\section{ABSTRACT}

Background The blood eosinophil count has been shown to be a promising biomarker for establishing personalised treatment strategies to reduce corticosteroid use, either inhaled or systemic, in chronic obstructive pulmonary disease (COPD). Eosinophil levels seem relatively stable over time in stable state, but little is known whether this is also true in subsequent severe acute exacerbations of COPD (AECOPD).

Aims and objectives To determine the stability in eosinophil categorisation between two subsequent severe AECOPDs employing frequently used cut-off levels. Methods During two subsequent severe AECOPDs, blood eosinophil counts were determined at admission to the hospital in 237 patients in the Cohort of Mortality and Inflammation in COPD Study. The following four cut-off levels were analysed: absolute counts of eosinophils $\geq 0.2 \times 10^{9} / \mathrm{L}$ ( 200 cells $/ \mu \mathrm{L}$ ) and $\geq 0.3 \times 10^{9} / \mathrm{L}$ (300 cells $/ \mu \mathrm{L}$ ) and relative eosinophil percentage of $\geq 2 \%$ and $\geq 3 \%$ of total leucocyte count. Categorisations were considered stable if during the second AECOPD their blood eosinophil status led to the same classification: eosinophilic or not.

Results Depending on the used cut-off, the overall stability in eosinophil categorisation varied between $70 \%$ and $85 \%$ during two subsequent AECOPDs. From patients who were eosinophilic at the first AECOPD, $34 \%-45 \%$ remained eosinophilic at the subsequent $A E C O P D$, while $9 \%-21 \%$ of patients being non-eosinophilic at the first AECOPD became eosinophilic at the subsequent AECOPD. Conclusions The eosinophil variability leads to category changes in subsequent AECOPDs, which limits the eosinophil categorisation stability. Therefore, measurement of eosinophils at each new exacerbation seems warranted.

\section{INTRODUCTION}

The use of corticosteroids, inhaled (ICS) or systemic, plays an important role in the maintenance treatment of chronic obstructive pulmonary disease (COPD) and in its associated acute exacerbations (AECOPD). ${ }^{1}$ Although beneficial for some, corticosteroids could be detrimental for others because of lack of efficacy combined with potential side effects such as pneumonia, hyperglycaemia, osteoporosis and venous thromboembolism. ${ }^{1-3}$ More and more focus is on how to

\section{Key messages}

\section{What is the key question?}

- What is the stability in eosinophil categorisation during subsequent severe exacerbations of chronic obstructive pulmonary disease (COPD)?

\section{What is the bottom line?}

- To reduce the unnecessary exposure to the negative effects of systemic steroids, eosinophil-guided strategies for systemic steroid use in acute exacerbations of COPD (AECOPD) are being studied. However, little is known about the stability of the blood eosinophil count during subsequent AECOPD and in particular the role of (recent) steroid use on this stability.

\section{Why read on?}

- In a large well-described COPD cohort, the eosinophil variability leads to category changes in subsequent AECOPDs. Therefore, measurement of eosinophils at each new exacerbation seems warranted.

select patients who probably will not benefit from corticosteroid use and in whom exposure to corticosteroids can safely be reduced or withheld.

In recent years, blood eosinophil count has shown to be a promising biomarker for establishing personalised treatment strategies to reduce corticosteroid use in COPD. ${ }^{2-6}$ Elevated blood eosinophil count, as a surrogate marker for sputum eosinophilia, is associated with better steroid responsiveness. ${ }^{78} \mathrm{In}$ stable-state COPD, it has become clear that the blood eosinophil count, in combination with clinical assessment, can be used to determine the treatment effect of ICS use. This has been incorporated in the Global Initiative for Chronic Obstructive Lung Disease (GOLD) 2019 report. $^{1}$

Two studies found evidence for an eosinophil-guided strategy for systemic steroid use also in acute exacerbations. ${ }^{23}$ Defining an AECOPD to be eosinophilic and defining the stability of an eosinophilic AECOPD phenotype over time are both vitally dependent 
on the chosen cut-off level and the expression of the eosinophil as an absolute or a relative count. The study of Bafadhel et al was in outpatients with a cut-off level of eosinophil count $\geq 2 \%$ of total leucocytes. The study of Sivapalan et al in inpatients used the cut-off of an absolute eosinophil count $\geq 0.3 \times 10^{9}$ cells $/ \mathrm{L}$. With their limitations, both studies showed that eosinophil-guided steroid use is non-inferior to standard systemic steroids during AECOPD and leads to a lower cumulative steroid exposure.

In stable-state COPD, eosinophil levels seem relatively stable over time, ${ }^{9-11}$ but whether this is also true in AECOPD is only knowledge from a small subset of patients $(n=67)$ in one study. ${ }^{12}$ Since different aetiologies exist for AECOPD, this stability may not be the case: elevated eosinophil levels may not be present every time. High stability would negate the need to determine blood eosinophils and accelerate the start of treatment. Low stability however implies that the eosinophil count has to be determined at every new AECOPD. This will add an extra barrier in the treatment of AECOPD, especially in the primary care setting and in COPD self-management.

Furthermore, it's possible that in daily practice, patients admitted to the hospital for an AECOPD are already on a course of systemic steroids or may have recently been on one. How large this impact is on the eosinophil level at presentation of an AECOPD and whether despite this steroid use patients can still have elevated eosinophil levels are both not well known.

So the aim of this study is to determine the stability in eosinophil categorisation during two subsequent severe (hospitalised) AECOPD and to study the influence of (recent) use of steroids on this stability. We will analyse this for four different cut-off levels including the two abovementioned ones and determine to what degree eosinophil variability, prior steroid use and expression of eosinophilia chosen lead to changes in ensuing treatment choice of systemic steroids.

To assess these questions, we used data of the large Cohort of Mortality and Inflammation in COPD (COMIC) Study with patients with well-defined COPD with a welldocumented medication history including steroid use.

\section{METHODS}

\section{Settings and study population}

This study was part of the COMIC Study, a singlecentre cohort study from Enschede, the Netherlands. From December 2005 to April 2010, 795 patients were included with a follow-up period of at least 3 years. The COMIC Study was approved by the hospital's medical ethical committee (METC Twente, number P05-49). All patients provided written informed consent. The COMIC Study started before the introduction of trial registries. Results from the cohort study have been published previously. ${ }^{13-15}$ For inclusion in the COMIC Study, patients had to meet the following criteria: (1) a clinical diagnosis of COPD according to the GOLD guidelines, (2) current or former smoker, (3) age $\geq 40$ years, (4) absence of any other active lung disease (eg, sarcoidosis) and (5) no maintenance therapy with antibiotics. For the current substudy, we performed an analysis in all patients with at least two subsequent severe AECOPDs leading to hospitalisation during follow-up.

\section{Patient and public involvement}

Patients and the public were not involved in the design and conduct of this study.

\section{Eosinophil counts and cut-off levels}

Blood eosinophil counts were determined at admission to the emergency room on the day of hospitalisation for a severe AECOPD. The eosinophil counts were determined with increments of $0.1 \times 10^{9}$ cells $/ \mathrm{L}(100$ cells $/ \mu \mathrm{L})$. The following four cut-off levels were analysed: absolute count of eosinophils $\geq 0.2 \times 10^{9}$ cells $/ \mathrm{L}$ (200 cells/ $\mu \mathrm{L})$ or $\geq 0.3 \times 10^{9}$ cells $/ \mathrm{L}(300$ cells $/ \mu \mathrm{L})$ and relative eosinophil percentage of $\geq 2 \%$ or $\geq 3 \%$ of total leucocyte count. Patients with an eosinophil count above the cutoff were categorised as eosinophilic and below as noneosinophilic.

\section{Outcomes}

The correlation between the two eosinophil counts during both AECOPDs was determined for both the absolute counts and the relative counts. Subsequently, to determine whether the variability of the two eosinophil counts during both AECOPDs led to a change in eosinophil categorisation, the overall eosinophil categorisation stability was established for all four different cut-off levels. This eosinophil stability was defined as the percentage of patients in whom their blood eosinophil count was categorised the same during the two subsequent AECOPDs.

\section{Role of steroid use on eosinophil categorisation stability}

To determine whether (recent) steroid use influenced the stability of eosinophil categorisation in subsequent severe AECOPD, the cohort was subdivided into three groups. Group 1 includes patients without steroid use before both subsequent AECOPDs. Group 2 includes patients with steroid use before both AECOPDs. Group 3 includes patients who used steroids either before the first AECOPD or before the second AECOPD. Recent steroid use was defined as the use of any systemic steroids in the last 2 weeks before the AECOPD.

\section{Effect steroid use on absolute eosinophil count}

To determine whether (recent) systemic steroid use influenced absolute eosinophil counts, the absolute eosinophil counts at the first AECOPD hospitalisation in the total cohort were compared between patients with or without prior steroid use before this hospitalisation. Furthermore, the effect of steroid use on the absolute 
eosinophil count within individuals was compared in group 3 in which paired eosinophil assessments were available per patient, one with and one without prior steroid use.

\section{Statistical analyses}

Continuous variables are expressed as mean with SD or median with IQR. Categorical variables are presented as counts with percentages. Baseline differences between the three groups of patients with or without prior steroid use were tested with either the ANalysis Of VAriance (ANOVA) or Kruskal-Wallis test for continuous variables and with $\chi^{2}$ or Fisher exact tests for categorical variables. Correlation of both the absolute and relative eosinophil counts between the two AECOPD hospitalisations was assessed by Spearman's rank correlation test. The effect of steroids on the absolute eosinophil count between patients with or without prior steroid use in the total cohort was assessed by the Mann-Whitney U test. The effect of steroids on the absolute eosinophil count within patients in group 3 with or without prior steroid was assessed by the Wilcoxon signed-rank test.

\section{RESULTS}

Of the 795 patients enrolled in the COMIC Study, 237 patients had at least two severe AECOPDs during follow-up. Of these 237 patients, 106 patients (45\%) did not use systemic steroids before the first and second severe AECOPDs (group 1); 32 patients (13\%) started a course of steroids before the first and second severe AECOPDs (group 2), while 99 patients (42\%) started steroids either before the first or the second severe AECOPD (group 3 ). The baseline characteristics of these 237 patients and the baseline characteristics of the patients within the three groups are displayed in table 1. Approximately $80 \%$ of the patients used ICSs. ${ }^{16}$ Patients in group 2 had a worse lung function, the duration between the two subsequent AECOPDs was shorter and they had more previous AECOPD.

Table 1 Baseline characteristics of the patients with two severe AECOPDs for the overall cohort and the three groups (patients without steroid use before both AECOPDs (group 1), patients with steroid use before both AECOPDs (group 2) and patients who used steroids either before the first AECOPD or before the second AECOPD (group 3))

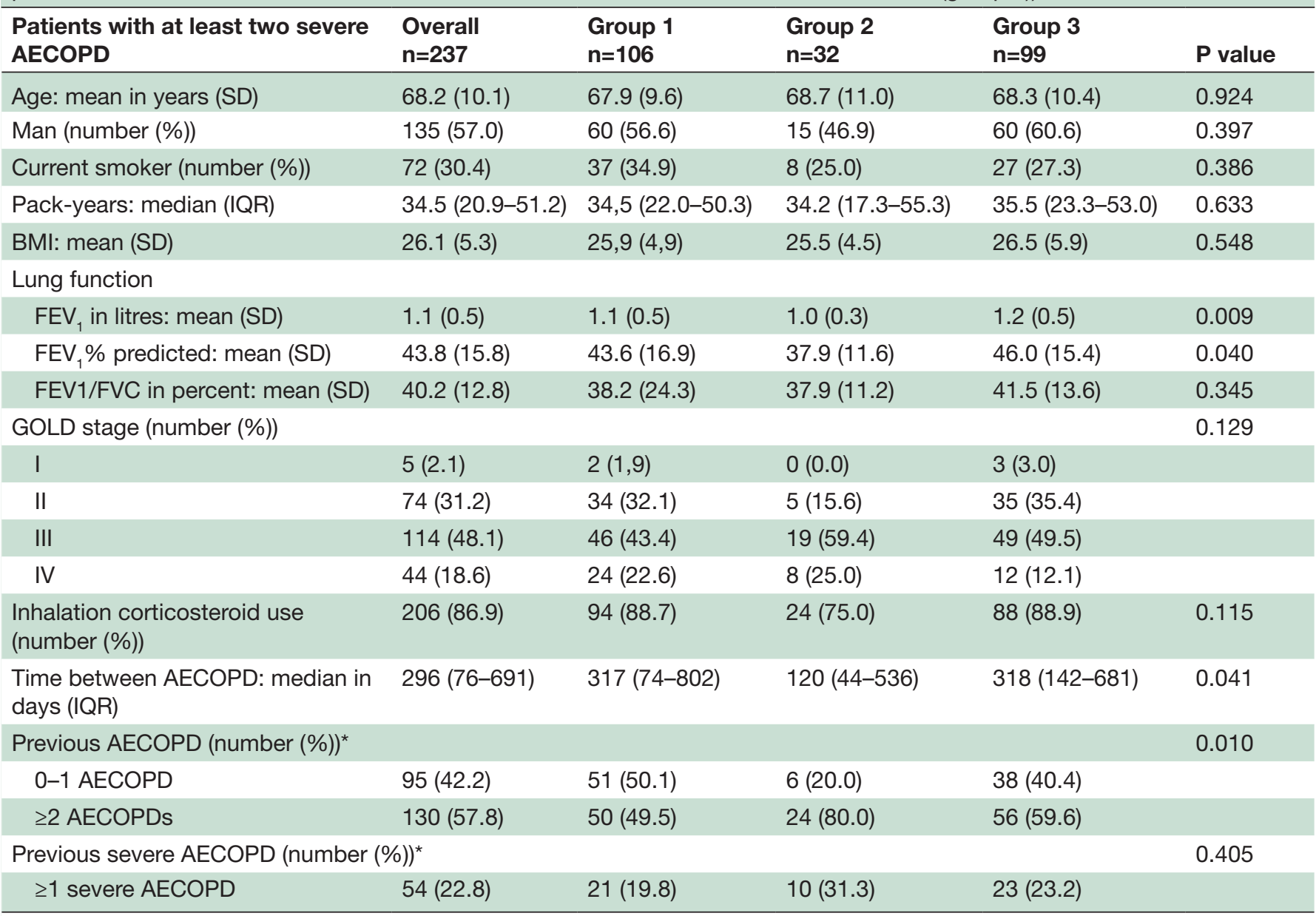

*In year before recruitment (missing data of 12 patients).

AECOPD, acute exacerbations of chronic obstructive pulmonary disease; BMI, body mass index; FEV , forced expiratory volume in $1 \mathrm{~s}$; FVC, Forced Vital Capacity; GOLD, Global Initiative for Chronic Obstructive Lung Disease.; 

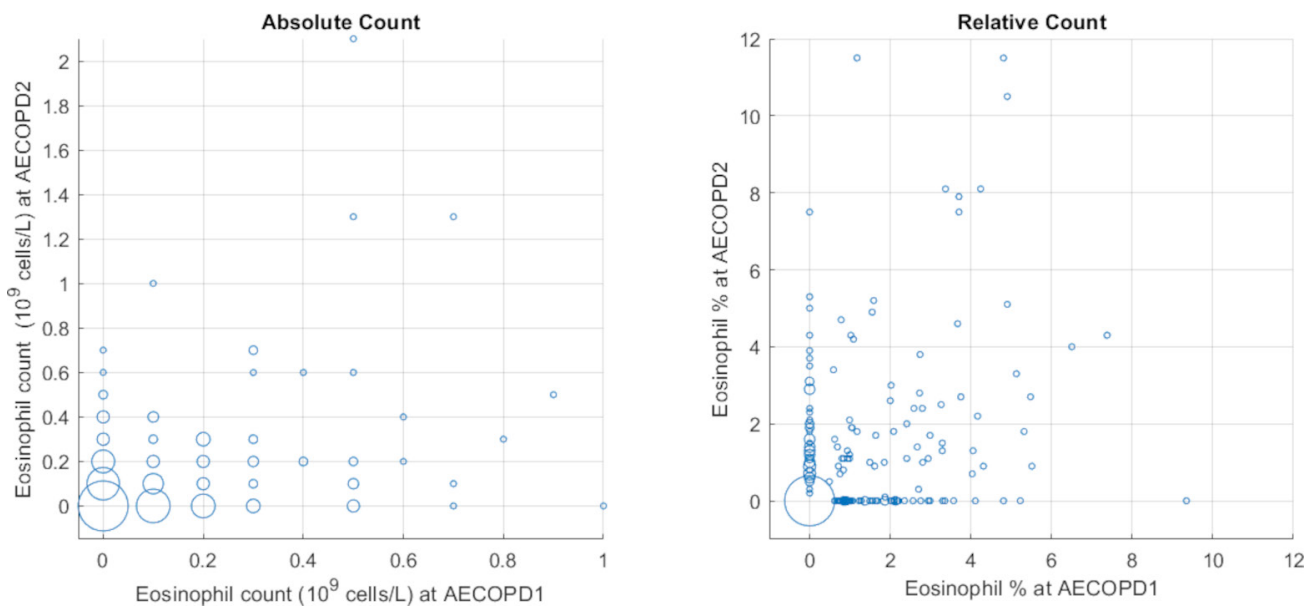

Figure 1 Scatter plot for the absolute and relative eosinophil counts measured at both severe AECOPDs. The size of the circle corresponds to the number of measurements with the smallest circle representing one measurement and the largest 66. Left: absolute count. Right: relative count. AECOPD, acute exacerbations of chronic obstructive pulmonary disease.

Figure 1 shows the scatter plots for the eosinophil count measured at both severe AECOPDs ( $\mathrm{rho}=0.19$, $\mathrm{p}=0.003$ for the absolute counts; rho $=0.22, \mathrm{p}=0.001$ for the relative counts).

The median absolute difference in eosinophil count between the two AECOPDs was $0.1 \times 10^{9}$ cells $/ \mathrm{L}$ (IQR $0.00-0.20)$. Stability of eosinophil categorisation over the two severe AECOPDs is shown in figure 2 for the overall cohort and the three groups.

In the total cohort, depending on the used cut-off, $12 \%-28 \%$ of the patients were eosinophilic at the first AECOPD, and $34 \%-45 \%$ of those remained eosinophilic at the second AECOPD. In total, $5 \%-13 \%$ of the patients were eosinophilic at both AECOPDs; 57\%-80\% of the patients were non-eosinophilic at both AECOPDs and 15\%-30\% changed from category at the second AECOPD. The eosinophil stability during the two AECOPDs was $70 \%-85 \%$.

In group $1,9 \%-28 \%$ of the patients were eosinophilic at the first AECOPD; $29 \%-50 \%$ of those remained eosinophilic at the second AECOPD. In total, 3\%-14\% of the patients were eosinophilic at both AECOPDs, while 58\%-78\% were non-eosinophilic at both AECOPDs, and 19\%-29\% changed from category at the second AECOPD. The eosinophil stability was $71 \%-81 \%$.
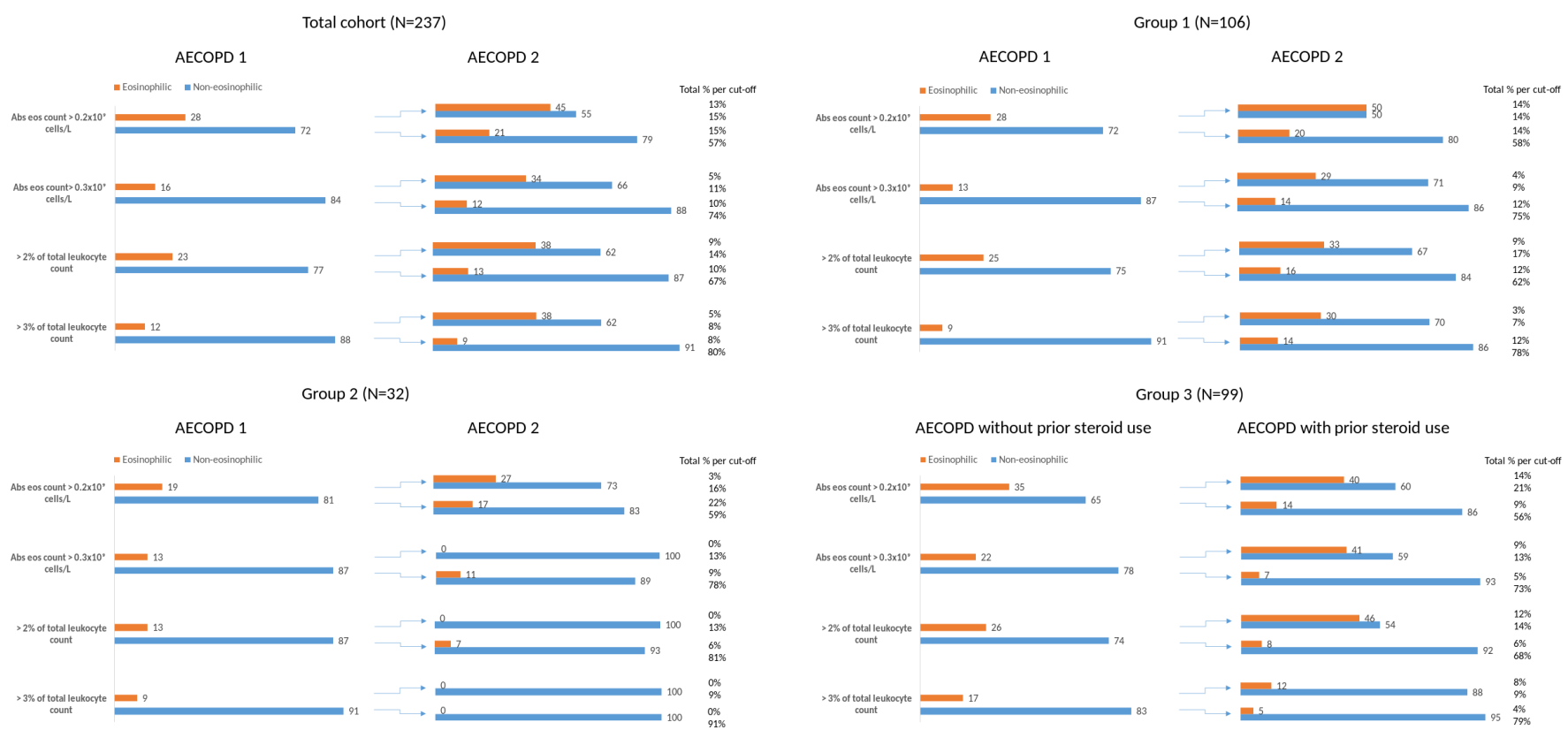

Figure 2 Stability of eosinophil categorisation over the two severe acute exacerbations of chronic obstructive pulmonary diseases (AECOPDs). Data presented separately for the total cohort, patients without steroid use before both AECOPDs (group 1), patients with steroid use before both AECOPDs (group 2) and patients who used steroids either before the first AECOPD or before the second AECOPD (group 3). The bars show the proportions of patients being categorised as eosinophilic or non-eosinophilic for the four used cut-offs at the first and second AECOPDs. 
In group 2, 9\%-19\% of the patients were eosinophilic at the first AECOPD, and $0 \%-27 \%$ of those remained eosinophilic at the second AECOPD. In total, $0 \%-3 \%$ of the patients were eosinophilic at both AECOPDs, while 59\%-91\% were non-eosinophilic at both AECOPDs, and 9\%-38\% changed from category at the second AECOPD. The eosinophil stability was $62 \%-91 \%$.

In group 3, 17\%-35\% of the patients were eosinophilic at the AECOPD without prior steroid use, and 12\%-46\% of them remained eosinophilic at the AECOPD with prior steroid use. In total, $8 \%-14 \%$ of the patients were eosinophilic at both AECOPDs, $56 \%-79 \%$ of the patients were non-eosinophilic at both AECOPDs and 13\%-30\% changed from category during both AECOPDs. The eosinophil stability was $70 \%-87 \%$.

\section{Effect of steroid use on absolute eosinophil count}

In the total cohort, the median eosinophil count in patients $(\mathrm{n}=145)$ who did not use steroids in the 2 weeks prior to the first severe AECOPD was $0.10 \times 10^{9}$ cells $/ \mathrm{L}$ (IQR 0.00-0.20), which was significantly higher than the eosinophil count in patients $(\mathrm{n}=92)$ who did use steroids in the 2 weeks prior to the first severe AECOPD (median eosinophil count $0.00 \times 10^{9}$ cells/L (IQR 0.00-0.10) $(\mathrm{p}=0.006))$. Of the 92 patients with systemic steroid use in the 2 weeks prior to hospitalisation for AECOPD, $37 \%$ still had an eosinophil count of $\geq 0.1 \times 10^{9}$ cells $/ \mathrm{L}$, and $10 \%-22 \%$ were still labelled as eosinophilic depending on the criterion used.

In group 3, including patients that had both a severe AECOPD with and without prior steroid use, the median eosinophil count determined at the AECOPD without prior steroid use was $0.10 \times 10^{9}$ cells/L (IQR $0.00-0.20$ ), which was significantly higher than the eosinophil count determined at the AECOPD with prior steroid use (median eosinophil count $0.00 \times 10^{9}$ cells/L (IQR $0.00-$ $0.10)(\mathrm{p}=0.016))$.

\section{DISCUSSION}

Our study shows that although a statistically significant correlation existed between eosinophil counts measured at subsequent severe AECOPDs, this correlation was very poor. The eosinophil counts were rather variable, and depending on the used cut-off and expression of the eosinophil, the overall stability in eosinophil categorisation varied between $70 \%$ and $85 \%$. This means that $15 \%-30 \%$ of the patients changed from category. Furthermore, systemic steroid use immediately prior to AECOPD lowered the eosinophil numbers, but did not normalise them and numerically had no relevant influence on the stability of the eosinophil categorisation.

Our results suggest that independent of which cutoff is used, when making a choice regarding systemic steroid treatment dependent on eosinophil numbers during a severe AECOPD, one cannot rely on the eosinophil count measured during an earlier severe AECOPD.
The eosinophil count has to be determined at every new severe AECOPD.

An important explanation for the variation in eosinophil count during subsequent severe AECOPDs leading to changes in eosinophil categorisation could lie in the variability in causes of AECOPD, which need not be the same at each exacerbation. Bafadhel et al described different biological and clinical COPD phenotypes during outpatient treated AECOPDs. ${ }^{17}$ Furthermore, Kolsum et al showed that patients with a bacterial infection during an AECOPD had a significant decrease in blood eosinophil count compared with stable state, while no changes were observed in patients without bacterial infection. ${ }^{8}$ In most cases, the exact pathobiology of eosinophils in blood is unclear. Analyses of RNA expression levels from sputum or, for instance, nasal epithelium and perhaps leucocyte activation markers in blood and/or sputum in relation to bacterial and viral load may help elucidate the origins of the eosinophilia. ${ }^{18} 19$

Although this study focuses on severe (hospitalised) AECOPD, we expect the same lack of stability in eosinophil categorisation in subsequent less severe AECOPDs, that is, without hospitalisation. Recently, among others, Lenferink et al reported the beneficial effects of selfmanagement interventions in COPD, including action plans for exacerbations, and they suggested to include this in COPD management. ${ }^{20}$ Eosinophil guidance in the treatment of AECOPD to become common practice would have major implications for COPD selfmanagement interventions as this should then lead to measuring eosinophils at the moment of AECOPD. As a consequence, healthcare providers of patients with COPD and patients with self-management action plans should have easy access to a (point of care) tool also in the outpatient clinic to determine their eosinophil count at AECOPD onset that should not delay treatment. In this study, patients with steroid use before both AECOPDs (group 2) had a worse lung function, more previous AECOPD and a shorter time between the two subsequent AECOPDs, possibly suggesting a more serious COPD. Maybe as a consequence, these patients possibly have COPD self-management action plans, which could explain why they were already on OCS on the day of laboratory testing for both of their AECOPDs.

The use of relative eosinophil percentages and the use of higher cut-off levels were both associated with higher overall stability in eosinophil categorisation. However, fewer AECOPDs will then be defined as eosinophilic. This will result in more patients who may be incorrectly withheld from steroids for their AECOPD when an eosinophilguided steroid strategy is used. The higher cut-off level used in the CORTICO-COP ${ }^{3}$ Study may therefore have influenced the observed, be it non-significant, higher 30-day readmission and death rates in the eosinophilguided arm. On the other hand, more patients had the advantage of not being exposed to the potential detrimental effects of steroids. Thus, the chosen cut-off level and the way of classifying eosinophils, relative versus 
absolute, are important factors to be considered when using eosinophil-guided strategies.

Another finding of this study is that also in COPD (recent) steroid use leads to significantly lower eosinophil counts measured at admission for their AECOPD, as expected. It is known, primarily from analyses in patients with asthma, that oral steroid use can lower blood eosinophil count significantly, even after one single dose, and that this suppression can last for more than 24 hours. ${ }^{21} 22$ Despite this lowering effect, significant eosinophilia persisted in many patients; steroid use did not have major influence on the eosinophil categorisation stability during subsequent AECOPD in our study; and still, about $10 \%-22 \%$ of the patients were categorised as eosinophilic when admitted to the hospital for AECOPD. This may contain important information. When patients need to be hospitalised for AECOPD, even though they had prior systemic steroid use, routine care often leads to higher and/or longer steroids. Where that may be justified in patients with high eosinophil counts, it might be less useful or even detrimental in patients without eosinophilia. ${ }^{3}$ In this latter CORTICO-COP Study, a strategy quite similar to this suggestion was used, resulting in a reduced duration of systemic corticosteroid exposure without significant difference in number of days alive and out of hospital.

In a study by Schumann et al, blood eosinophil counts were longitudinal collected in patients with COPD in stable state and in AECOPD. ${ }^{12}$ During severe exacerbations, up to one-half of the patients had discordant or variable blood eosinophil levels, dependent on which cut-off was used, which is even worse than our results. These analyses were however in a small subset of patients $(n=67)$, and no information about previous steroid use was available. This is contrary to our study in which being able to study the influence of (recent) steroid use in a large group of patients with COPD is the major strength of our analysis.

A limitation of our study is however that although (recent) steroid use was defined by the dispensing of the medication at the pharmacy, we cannot determine with certainty that the patient actually used the medication or if perhaps a patient used steroids that were dispensed longer ( $>14$ days) before the severe AECOPD.

Since differences in steroid use prior to the two included severe AECOPDs could have influenced the stability of eosinophil categorisation, we also performed sensitivity analyses in three subgroups of the cohort (based on prior steroid use). These sensitivity analyses showed that (recent) steroid use (within 2 weeks before the AECOPD) or not in fact has no relevant influence on this stability of eosinophil categorisation. Additionally, we studied recent steroid use defined as the start of OCS within one week before the AECOPD (see online supplemental index figure 1) indicating definite OCS use during laboratory testing. Numerically, this had no effect on stability of eosinophil categorisation, although groups 2 and 3 became much smaller now.
So far, there is no consensus on an acceptable degree of stability of eosinophil categorisation, so the results of our study are open for debate. We strongly believe, as our results show, that if $\geq 55 \%$ of the patients categorised as eosinophilic were not categorised as eosinophilic at the subsequent AECOPD, an eosinophil-guided strategy for steroid use for AECOPD can only be incorporated if the eosinophil count will be determined at every new AECOPD. As with any binary categorisation, small changes around the threshold have major impact on the category. Maybe, just as in stable state, one should not use just one cut-off but see the eosinophil count more as a continuous variable and use, for instance, two cutoff levels. This results in three categories in which the high (eosinophilic group) level should receive steroids and the low (non-eosinophilic group) level should not receive steroids, and for the intermediate group, steroid use should be individualised using other (clinical or historical) parameters. Of course, this strategy should be prospectively studied as well.

Finally, the eosinophil count was established with increments of $0.1 \times 10^{9}$ cells $/ \mathrm{L}$. Therefore, another frequently used cut-off, that is, $0.15 \times 10^{9}$ cells $/ \mathrm{L}$, could not be used in our analyses. However, this cut-off has mainly been used in stable state and was not one of the cut-offs used for steroid use in AECOPD. We have performed additional analyses for the $\geq 0.10 \times 10^{9}$ cells $/ \mathrm{L}$ cut-off (see online supplemental index figure 2), which is used in the GOLD guideline for assessing ICS use in stable state. This resulted in lower eosinophil categorisation stability, which is in line with our findings that lower cut-offs are associated with lower overall eosinophil categorisation stability.

Time between the two AECOPDs (using the median) was not associated with the categorisation stability in the overall cohort tested for all the cut-offs (data not shown). It would be interesting for further research to study factors that could predict this eosinophil categorisation stability. Perhaps in subgroups of patients (ie, persistent smokers and elderly patients), this stability may be better or worse. A relevant factor should then also be the aetiology of the AECOPD.

In summary, eosinophil categorisation varies in subsequent severe AECOPDs. This variability leads to category changes in subsequent AECOPDs, which limits the eosinophil categorisation stability. Although (recent) steroid use was associated with lower baseline eosinophil count, numerically, it has no relevant influence on the categorisation stability. Until we understand more about the causes of eosinophilia, quick-access, point-of-care measurements at each new exacerbation seem warranted.

Contributors EC contributed to the study design, data collection, statistical analysis and writing of the manuscript; he is the guarantor of the manuscript and takes responsibility for the integrity of the data and the accuracy of the data analysis. JvdP and MB-K contributed to the study design, statistical analysis and writing of the manuscript. PvdV contributed to the writing of the manuscript. HAMK contributed to the study design and writing of the manuscript. 
Funding The COMIC Study was supported by the Department of Pulmonology, Medisch Spectrum Twente, and by an unrestricted grant from GlaxoSmithKline. The sponsor had no role in the design of the study, the collection and analysis of the data or the preparation of the manuscript.

Competing interests None declared.

Patient consent for publication Not required.

Ethics approval The COMIC Study was approved by the hospital's medical ethical committee (METC Twente, number P05-49).

Provenance and peer review Not commissioned; externally peer reviewed.

Data availability statement Data are available on reasonable request. All data relevant to the study are included in the article or uploaded as supplementary information.

Supplemental material This content has been supplied by the author(s). It has not been vetted by BMJ Publishing Group Limited (BMJ) and may not have been peer-reviewed. Any opinions or recommendations discussed are solely those of the author(s) and are not endorsed by BMJ. BMJ disclaims all liability and responsibility arising from any reliance placed on the content. Where the content includes any translated material, BMJ does not warrant the accuracy and reliability of the translations (including but not limited to local regulations, clinical guidelines, terminology, drug names and drug dosages), and is not responsible for any error and/or omissions arising from translation and adaptation or otherwise.

Open access This is an open access article distributed in accordance with the Creative Commons Attribution Non Commercial (CC BY-NC 4.0) license, which permits others to distribute, remix, adapt, build upon this work non-commercially, and license their derivative works on different terms, provided the original work is properly cited, appropriate credit is given, any changes made indicated, and the use is non-commercial. See: http://creativecommons.org/licenses/by-nc/4.0/.

\section{REFERENCES}

1 Global Initiative for Chronic Obstructive Lung Disease. 2020 Global strategy for the prevention, diagnosis and management of COPD, 2020. Available: https://goldcopd.org/gold-reports/

2 Bafadhel M, McKenna S, Terry S, et al. Blood eosinophils to direct corticosteroid treatment of exacerbations of chronic obstructive pulmonary disease: a randomized placebo-controlled trial. $\mathrm{Am} J$ Respir Crit Care Med 2012;186:48-55.

3 Sivapalan P, Lapperre TS, Janner J, et al. Eosinophil-guided corticosteroid therapy in patients admitted to hospital with COPD exacerbation (CORTICO-COP): a multicentre, randomised, controlled, open-label, non-inferiority trial. Lancet Respir Med 2019;7:699-709.

4 Bafadhel M, Davies L, Calverley PMA, et al. Blood eosinophil guided prednisolone therapy for exacerbations of COPD: a further analysis. Eur Respir J 2014;44:789-91.

5 Bafadhel M, Pavord ID, Russell REK. Eosinophils in COPD: just another biomarker? Lancet Respir Med 2017;5:747-59.

6 Bafadhel M, Peterson S, De Blas MA, et al. Predictors of exacerbation risk and response to budesonide in patients with chronic obstructive pulmonary disease: a post-hoc analysis of three randomised trials. Lancet Respir Med 2018;6:117-26.

7 Brightling CE, Monteiro W, Ward R, et al. Sputum eosinophilia and short-term response to prednisolone in chronic obstructive pulmonary disease: a randomised controlled trial. Lancet 2000;356:1480-5.

8 Kolsum U, Donaldson GC, Singh R, et al. Blood and sputum eosinophils in COPD; relationship with bacterial load. Respir Res 2017;18:88.

9 Brusselle G, Pavord ID, Landis S, et al. Blood eosinophil levels as a biomarker in COPD. Respir Med 2018;138:21-31.

10 Oshagbemi OA, Burden AM, Braeken DCW, et al. Stability of blood eosinophils in patients with chronic obstructive pulmonary disease and in control subjects, and the impact of sex, age, smoking, and baseline counts. Am J Respir Crit Care Med 2017;195:1402-4.

11 Southworth T, Beech G, Foden P, et al. The reproducibility of COPD blood eosinophil counts. Eur Respir J 2018;52. doi:10.1183/13993003.00427-2018. [Epub ahead of print: 2707 2018].

12 Schumann DM, Tamm M, Kostikas K, et al. Stability of the blood eosinophilic phenotype in stable and exacerbated COPD. Chest 2019;156:456-65.

13 Brusse-Keizer M, Klatte M, Zuur-Telgen M, et al. Comparing the 2007 and 2011 gold classifications as predictors of all-cause mortality and morbidity in COPD. COPD 2017;14:7-14.

14 Citgez E, van der Palen J, Koehorst-Ter Huurne K, et al. Statins and morbidity and mortality in COPD in the COMIC study: a prospective COPD cohort study. BMJ Open Respir Res 2016;3:e000142.

15 Citgez E, Zuur-Telgen M, van der Palen J, et al. Stable-State Midrange Proadrenomedullin Is Associated With Severe Exacerbations in COPD. Chest 2018;154:51-7.

16 Koehorst-Ter Huurne K, Kort S, van der Palen J, et al. Quality of life and adherence to inhaled corticosteroids and tiotropium in COPD are related. Int J Chron Obstruct Pulmon Dis 2016;11:1679-88.

17 Bafadhel M, McKenna S, Terry S, et al. Acute exacerbations of chronic obstructive pulmonary disease: identification of biologic clusters and their biomarkers. Am J Respir Crit Care Med 2011;184:662-71.

18 Christenson SA, Steiling K, van den Berge M, et al. AsthmaCOPD overlap. clinical relevance of genomic signatures of type 2 inflammation in chronic obstructive pulmonary disease. Am J Respir Crit Care Med 2015;191:758-66.

19 Imkamp K, Berg M, Vermeulen CJ, et al. Nasal epithelium as a proxy for bronchial epithelium for smoking-induced gene expression and expression quantitative trait loci. J Allergy Clin Immunol 2018;142:314-7.

20 Lenferink A, van der Palen J, van der Valk PDLPM, et al. Exacerbation action plans for patients with COPD and comorbidities: a randomised controlled trial. Eur Respir J 2019;54 doi:10.1183/13993003.02134-2018. [Epub ahead of print: 0711 2019].

21 Berthon BS, Gibson PG, Wood LG, et al. A sputum gene expression signature predicts oral corticosteroid response in asthma. Eur Respir $J$ 2017;49. doi:10.1183/13993003.00180-2017. [Epub ahead of print: 2906 2017].

22 Gibson PG. Variability of blood eosinophils as a biomarker in asthma and COPD. Respirology 2018;23:12-13. 